hep-th/9901020

CERN-TH/98-412

CPHT-S707.1298

\title{
Higher Derivative Couplings and Heterotic-Type I Duality in Eight Dimensions
}

\author{
K. Förger ${ }^{1}$ and S. Stieberger ${ }^{2}$ \\ 1 Centre de Physique Théorique \\ École Polytechnique \\ F-91128 Palaiseau Cedex, FRANCE \\ 2 CERN, Theory Division \\ CH-1211 Geneva 23, SWITZERLAND
}

\begin{abstract}
We calculate $F^{4}$ and $R^{4} T^{4 g-4}$ couplings in $d=8$ heterotic and type I string vacua (with gauge and graviphoton field strengths $F, T$, and Riemann curvature $R$ ). The holomorphic piece $F_{g}$ of the heterotic one-loop coupling $R^{4} T^{4 g-4}$ is given by a polylogarithm of index $5-4 g$ and encodes the counting of genus $g$ curves with $g$ nodes on the $K 3$ of the dual $F$-theory side. We present closed expressions for world-sheet $\tau$-integrals with an arbitrary number of lattice vector insertions. Furthermore we verify that the corresponding heterotic one-loop couplings sum up perturbative open string and non-perturbative D-string contributions on the type I side. Finally we discuss a type I one-loop correction to the $R^{2}$ term.
\end{abstract}

$12 / 98$ 


\section{Introduction}

Heterotic $S O(32) /$ type I duality relates two string theories in ten dimensions [1] [2]. A field redefinition transforms the low energy effective action of the heterotic string into the one of the type I string. This duality map is valid for second derivative terms as well as for higher derivative terms containing four or eight derivatives that can be arranged into superinvariants [3]. The low energy effective actions of the heterotic and type I string theory are related by the following field redefinitions in $d=10$ [2] [3]:

$$
G_{\mu \nu}^{I}=\lambda^{I} G_{\mu \nu}^{\text {het }} \quad \lambda^{I}=\frac{1}{\lambda^{\text {het }}} \quad B_{\mu \nu}^{R, I}=B_{\mu \nu}^{N S, \text { het }} \quad A_{\mu}^{a, I}=A_{\mu}^{a, \text { het }}
$$

where $a=1, \ldots, 16$ labels $S O(32)$ gauge indices, $G_{\mu \nu}$ is the metric and $B_{\mu \nu}^{N S / R}$ the antisymmetric tensor in the $N S$ or $R$ sector and $\lambda^{I}=e^{\phi^{(10)}}$ is the ten dimensional type I coupling. Compactifying to lower dimensions modifies these relations due to the dependence on the volume of the compactification manifold [4]

$$
\phi_{\text {het }}^{d}=\frac{6-d}{4} \phi_{I}^{d}-\frac{d-2}{16} \ln \operatorname{det} G_{I}
$$

where $G_{I}$ is the internal metric in the type I string frame. This relation shows that the critical dimension $d=6$ separates a strong-weak coupling duality from a weak-weak coupling duality.

Toroidal compactification down to eight dimensions gives rise to 4 Abelian gauge fields, corresponding to components of $G_{\mu I}$ and $B_{\mu I}$, where $\mu$ is the space-time index and $I$ labels the compact directions. The four massless scalar fields from $G_{I J}$ and $B_{I J}$ combine into two complex scalars $T, U$ and parametrize the moduli space $\frac{O(2,2)}{O(2) \times O(2)}$ of the torus $T^{2}$ (if no Wilson lines are switched on). In the type I theory the $U(1)^{2} \times U(1)^{2}$ gauge group is reduced to a diagonal $U(1)^{2}$, due to the twist operator $\Omega$ [5]. Similarly only two of the four massless scalar fields survive the projection.

Besides a comparison of the BPS spectrum of the heterotic and type I string nontrivial checks can be done for the BPS-saturated $F^{4}$ and $R^{4}$ couplings; see e.g. [6] [7] [8] [9] and [10] [11] for gauge couplings in the context of heterotic/F-theory duality. The special feature of these couplings is that -on the heterotic side- they are protected from higher than one-loop corrections in the effective action because of supersymmetry. Thus they can be exactly calculated. These threshold corrections translate to perturbative open string amplitudes and non-perturbative BPS D1-instanton corrections on the type I side. 
Therefore by studying various examples of couplings in any dimension one gains insight into the rules of computing non-perturbative $D$-brane contributions. It is the aim of this article to pursue these ideas further. The subject are eight-dimensionall 1 heterotic and type I string vacua coming from toroidal compactification of ten dimensions. We calculate various couplings of higher space-time derivatives and higher order in $\alpha^{\prime}$ for both heterotic (section 2) and type I string vacua (section 3) and compare them: In section 2.1 we review the heterotic $R^{4}$ couplings, since they capture a part of the structure of the $F^{4}$ couplings which originate in the torus compactification to eight dimensions. These couplings are calculated in section 2.2. and 2.3. The latter section contains also closed expressions for world-sheet $\tau$-integrals with an arbitrary number of lattice vector insertions. In section 2.4. we calculate the higher order corrections $R^{4} T^{4 g-4}$, which have a nice interpretation on the dual $F$-theory side. The type I calculation of these couplings are presented in sections 3.1-3.3. In section 3.4. we show that, a part of the heterotic $R^{4} T^{4 g-4}$ couplings has an intriguing physical interpretation in term of the dual type I string picture as $D$-instanton sum. Finally in section 3.5. we find a one-loop $R^{2}$ corrections in eight-dimensional type I string theory and give its explanation on the heterotic side.

\section{Heterotic string in eight dimensions}

In this section we consider $T^{2}$ compactifications of the heterotic string. The heterotic dilaton in eight dimensions is a real scalar in the supergravity multiplet [13]. Therefore, it is believed that there exist no higher than one-loop perturbative corrections, since by supersymmetry they would also affect the Einstein term. At string one-loop, higher derivative couplings such as $F^{4},\left(F^{2}\right)^{2}, R^{4}$ and $\left(R^{2}\right)^{2}$ and their respective anomaly-cancelling $C P$ odd parts receive corrections in the effective string action. These constant contributions are highly fixed by ten-dimensional anomaly cancellation arguments. In Green-Schwarz formalism they are calculated by (almost) holomorphic one loop string amplitudes, whose minimal number of external legs is fixed by saturating fermionic zero modes. The result for the amplitudes is summarized by the worldsheet $\tau$ integral over a weight zero almost holomorphic function which is related to the elliptic genus [14][15]. After compactification on $T^{2}$ these corrections become moduli dependent functions. It is believed that there are no space-time instanton effects, since the only supersymmetric soliton, the NS five-brane, cannot be wrapped around the torus. On the other hand, the non-anomaly related coupling to $J_{0}=t_{8} t_{8} R^{4}-\frac{1}{8} \epsilon_{10} \epsilon_{10} R^{4}$ may receive higher perturbative as well as non-perturbative corrections.

1 Other interesting aspects of $D=8$ theories have been recently discussed in [12]. 


\subsection{Ten dimensional origin}

Let us briefly review the four graviton amplitude. The graviton vertex operator can be read off from the background field expansion of the heterotic sigma model on the worldsheet torus 15 16 and reads in the Green-Schwarz formalism

$$
V_{\mathrm{gr}}=-\frac{1}{3} R_{\mu \rho \nu \sigma}: \bar{\partial} X^{\mu} X^{\rho}\left(\partial X^{\nu} X^{\sigma}+\frac{3}{8} \gamma_{a b}^{\nu \sigma} S^{a} S^{b}\right):
$$

where $X^{\mu}$ are bosonic fields with $\mu=1 \ldots 8$ and $S^{a}$ with $a=1 \ldots 8$ their supersymmetric partners. The four-point graviton correlation function is $⿴$

$$
\left\langle\prod_{i=1}^{4} V_{\mathrm{gr}}^{i}\right\rangle=\frac{1}{2^{4}} t^{\nu_{1} \sigma_{1} \nu_{2} \sigma_{2} \nu_{3} \sigma_{3} \nu_{4} \sigma_{4}} \prod_{i=1}^{4} R_{\mu_{i} \rho_{i} \nu_{i} \sigma_{i}}\left\langle\prod_{i=1}^{4}: \bar{\partial} X_{i}^{\mu_{i}} X_{i}^{\rho_{i}}:\right\rangle
$$

The tensor $t_{8} \equiv t^{i j k l m n p q}=\frac{\gamma_{a b}^{i j}}{4} \frac{\gamma_{c d}^{k l}}{4} \frac{\gamma_{e f}^{m n}}{4} \frac{\gamma_{g h}^{p q}}{4} \epsilon^{a b c d e f g h}$ is defined e.g. as in [17]. In the path integral formalism it arises after integration over fermionic zero modes.

Depending on different ways of contracting bosonic fields the worldsheet integration results in two different couplings [14]. Contractions which are associated to permutation cycles (1)(234) give rise to the $\operatorname{Tr} R^{4}$ coupling $\bar{G}_{4}$, whereas contractions corresponding to permutation cycles $(12)(34)$ result in the $\left(\operatorname{Tr} R^{2}\right)^{2}$ coupling $\hat{\bar{G}}_{2}^{2}$. The Eisenstein functions $\hat{G}_{2 k}$ of weight $2 k$ can be interpreted as 'gravitational' charge insertions. 3

The pure gravitational part of the effective action is [14]

$$
S_{\text {eff }}^{1-\text { loop }}=-\left.\mathcal{N} \int \frac{d^{2} \tau}{\tau_{2}^{2}} \tilde{\mathcal{A}}(\bar{q}, R)\right|_{8-\text { form }}
$$

where $\mathcal{N}=\frac{V^{(10)}}{2^{10} \pi^{6}}$ is the normalization factor including the uncompactified volume and with the elliptic genus

$$
\mathcal{A}(\bar{q}, 0, R)=\exp \left[\sum_{k=1}^{\infty} \frac{1}{4 k} \frac{1}{(2 \pi i)^{2 k}} \operatorname{Tr}\left(\frac{i R}{2 \pi}\right)^{2 k} \bar{G}_{2 k}(\bar{\tau})\right] \frac{\bar{E}_{4}^{2}}{\bar{\eta}^{24}}
$$

${ }^{2}$ Using the vertex operator $V_{\mathrm{gr}}=\epsilon_{\mu \nu}: \bar{\partial} X^{\mu}\left(\partial X^{\nu}-\frac{\gamma_{a b}^{\rho \nu}}{4} k_{\rho} S^{a} S^{b}\right) e^{i k \cdot X}:$ the $\mathcal{O}\left(k^{4}\right)$ piece of the four point graviton amplitude produces the one-loop $\operatorname{Tr} R^{2}$ coupling with bosonic correlator $\int \prod_{i=1}^{4} d^{2} z_{i}\left\langle\prod_{i=1}^{4} \bar{\partial} X^{\mu_{i}}\right\rangle$, which gives no contribution after $z$-integration [14].

3 The relation between different normalized Eisenstein functions is $G_{2 k}=-\frac{(2 \pi i)^{2 k}}{(2 k) !} B_{2 k} E_{2 k}=$ $2 \zeta(2 k) E_{2 k}$, where $B_{2 k}$ are the Bernoulli numbers. 
where the last term is the $E_{8} \times E_{8}$ light-cone partition function $\mathcal{A}(\bar{q})=\frac{1}{\bar{\eta}^{24}} \sum_{P \in \Gamma_{16,0}} q^{P^{2} / 2}$, which is a modular form of weight -4 . Since the effective action has to be modular invariant the elliptic genus is replaced by its regularized version $\tilde{\mathcal{A}}(q, R)$ depending on the non-holomorphic function $\hat{G}_{2}$ instead of $G_{2}$.

The action (2.3) has also to be supplemented with the corresponding GS-counterterm which will provide us the $C P$-odd part of our amplitudes and gives the eight derivatives gravitational superinvariants $I_{3}, I_{4}[3]$.

\section{2. $F^{4}$ heterotic couplings}

For toroidal compactification the amplitudes include a lattice sum, which arises from integration over zero modes of compactified bosons. In addition to the gauge group $E_{8} \times E_{8}$ or $S O(32)$ (without additional Wilson lines) there are extra $U(1)$ gauge groups. Here we will mainly concentrate on these $U(1)$ gauge couplings and consider the case without and with Wilson lines separately. The internal gauge boson vertex operator can be read off from the $\sigma$ model action. Writing the internal metric as

$$
G_{I J}=\frac{T_{2}}{U_{2}}\left(\begin{array}{cc}
1 & U_{1} \\
U_{1} & |U|^{2}
\end{array}\right)=\epsilon_{(I} \bar{\epsilon}_{J)}
$$

with $\epsilon_{I}=\sqrt{\frac{T_{2}}{U_{2}}}(1, U)$ and $\bar{\epsilon}_{J}=\sqrt{\frac{T_{2}}{U_{2}}}(1, \bar{U})$ the vertex operator for $G_{\mu I}$ gauge bosons in a background gauge $\epsilon_{\mu} e^{i k \cdot X}=-\frac{1}{2} F_{\mu \rho} X^{\rho}$ with $F_{\mu \nu}=$ const. in Green-Schwarz formalism is:

$$
V_{\text {gauge }}=\frac{i \pi}{\tau_{2}} F_{\mu \nu}^{A} \bar{Q}_{R}^{A}:\left(\partial X^{\mu} X^{\nu}-\frac{1}{4} S^{a} \gamma_{a b}^{\mu \nu} S^{b}\right):
$$

where $A=1,2$ labels different $U(1)$ charges

$$
\begin{aligned}
& Q_{R}^{1} \equiv \bar{Q}_{R}=\epsilon_{I} \bar{\partial} X^{I}=\sqrt{\frac{T_{2}}{U_{2}}}(1, U) A\left(\begin{array}{c}
\tau \\
1
\end{array}\right), \\
& Q_{R}^{2} \equiv Q_{R}=\bar{\epsilon}_{I} \bar{\partial} X^{I}=\sqrt{\frac{T_{2}}{U_{2}}}(1, \bar{U}) A\left(\begin{array}{l}
\tau \\
1
\end{array}\right)
\end{aligned}
$$

where $A=\left(\begin{array}{l}n_{1}-l_{1} \\ n_{2}-l_{2}\end{array}\right)$ is a $G L(2, \mathbf{Z})$ matrix. The complex structure and the Kähler structure of the torus are defined in terms of the metric and NS-NS antisymmetric tensor as $U=$ $U_{1}+i U_{2}=\left(G_{12}+i \sqrt{\operatorname{det} G}\right) / G_{11}$ and $T=T_{1}+i T_{2}=2(b+i \sqrt{\operatorname{det} G})$, respectively.

Inspection of the four point amplitude $\left\langle\prod_{i=1}^{4} V_{\text {gauge }}^{A_{i}}\right\rangle$ shows that replacing $R_{\mu \rho \nu \sigma} \bar{\partial} X^{\mu} X^{\rho}$ by $F_{\mu \nu, A} Q^{A}$ in (2.2) turns the four graviton amplitude into the four gauge boson amplitude 
with bosonic correlator $\left\langle\prod_{i=1}^{4} Q_{R, i}^{A}\right\rangle_{\text {int }}$. This correlation function now includes a sum over winding modes $\mathbf{n}$ and $\mathbf{l}$ of the $\Gamma_{(2,2)}$ lattice.

The effective action depending on these couplings can be expressed as:

$$
\begin{aligned}
S_{1-\text { loop }}^{\text {het }} & =\frac{V^{(8)} T_{2}}{2^{8} \pi^{4}} \int \frac{d^{2} \tau}{\tau_{2}^{2}} \sum_{1, \mathbf{n} \in \mathbf{Z}} e^{-\frac{2 \pi}{\tau_{2}}\left(n^{I} \tau-l^{I}\right)(G+B)_{I J}\left(n^{J} \bar{\tau}-l^{J}\right)} \\
& \times \int D S_{0}^{a} e^{-\pi / \tau_{2} F_{\mu \nu, A} Q_{R}^{A} R_{0}^{\mu \nu}} \overline{\mathcal{A}}(\bar{q})
\end{aligned}
$$

where $R_{0}^{\mu \nu}=\frac{1}{4} S_{0}^{a} \gamma_{a b}^{\mu \nu} S_{0}^{b}$.

Berezin integration thus produces the following terms in the effective action

$$
S_{1-\text { loop }}^{\text {het }}=\frac{V^{(8)} T_{2}}{2^{8} \pi^{4}} \Delta_{F_{1}^{4-q} F_{2}^{q}} t_{8} F_{1}^{4-q} F_{2}^{q}
$$

for $q=0, \ldots, 4$ with coupling

$$
\begin{aligned}
\Delta_{F_{1}^{4-q} F_{2}^{q}} & =\frac{\partial^{4-q}}{\partial \Lambda_{1}^{4-q}} \frac{\partial^{q}}{\partial \Lambda_{2}{ }^{q}} \times \\
& \times\left.\int \frac{d^{2} \tau}{\tau_{2}^{2}} \sum_{A \in G L(2, \mathbf{Z})} e^{2 \pi i T \operatorname{det} A-\frac{\pi T_{2}}{\tau_{2} U_{2}}\left|(1, U) A\left({ }_{-1}^{\tau}\right)\right|^{2}} e^{-\Lambda_{A} \frac{\pi}{\tau_{2}} Q_{R}^{A}} \overline{\mathcal{A}}(\bar{q})\right|_{\Lambda=0} .
\end{aligned}
$$

The gravitational charges $G_{2 k}$ are now replaced by $\prod_{i=1}^{4} \frac{\pi}{\tau_{2}} Q^{A_{R, i}}$ which transforms under $S L(2, \mathbf{Z})_{\tau}$ as a modular function of weight 4 .

Poisson resummation turns the sum over winding modes $\mathbf{l}, \mathbf{n}$ into a sum over momenta $\mathbf{m}$ and windings $\mathbf{n}$. The partition function now contains Narain momenta insertions

$$
\begin{aligned}
& p_{R}=\frac{1}{\sqrt{2 T_{2} U_{2}}}\left[m_{1}+m_{2} \bar{U}+T\left(n_{1}+n_{2} \bar{U}\right)\right] \\
& p_{L}=\frac{1}{\sqrt{2 T_{2} U_{2}}}\left[m_{1}+m_{2} \bar{U}+\bar{T}\left(n_{1}+n_{2} \bar{U}\right)\right] .
\end{aligned}
$$

The different couplings $\Delta_{F_{1}^{4-q} F_{2}^{q}}$ include charge insertions $\left(\bar{Q}_{R}\right)^{4-q}\left(Q_{R}\right)^{q}$. From (2.10) the $\operatorname{Tr} F_{1}^{4-q} F_{2}^{q}$ coupling reads:

$$
\Delta_{F_{1}^{4-q} F_{2}^{q}}=\pi^{4} \int \frac{d^{2} \tau}{\tau_{2}^{6}} \sum_{\substack{n_{1}, n_{2} \\ l_{1}, l_{2}}}\left(\bar{Q}_{R}\right)^{4-q}\left(Q_{R}\right)^{q} e^{-2 \pi i \bar{T} \operatorname{det} A} e^{\frac{-\pi T_{2}}{\tau_{2} U_{2}}\left|n_{1} \tau+n_{2} U \tau-U l_{1}+l_{2}\right|^{2}} \frac{\bar{E}_{4}^{2}}{\bar{\eta}^{24}} .
$$

4 Supersymmetry relates even to odd spin structures. Since periodic Green-Schwarz fields $S^{a}$ are mapped to periodic NSR field $\psi^{i}, C P$-odd correlation functions of NSR currents are equivalent to $C P$-even correlation functions of Green-Schwarz currents due to a Riemann identity [15]. 
This $\tau$-integral has been solved in [10] by the method of orbit decomposition [18] and techniques developed in [19]. It has been shown in [10] that these four point functions (2.12) are related to a holomorphic prepotential $\mathcal{G}(T, U)$ which transform as a modular function of weight $(-4,-4)$ under $S L(2, \mathbf{Z})_{T} \times S L(2, \mathbf{Z})_{U}$ duality. This is similar to vector multiplet sector of $\mathrm{N}=2$ supersymmetric string vacua in four dimensions. They arrange as fourth order covariant derivatives

$$
\Delta_{F_{1}^{4-q} F_{2}^{q}}=16 \pi i\left(\frac{T_{2}}{U_{2}}\right)^{2-q} D_{T}^{4-q} D_{U}^{q} \mathcal{G}-16 \pi i\left(\frac{U_{2}}{T_{2}}\right)^{2-q} \bar{D}_{\bar{T}}^{q} \bar{D}_{\bar{U}}^{4-q} \overline{\mathcal{G}}
$$

of the prepotential $\mathcal{G}[\mathbb{[ 1 0 ]}$

$$
\mathcal{G}(T, U)=-\frac{i c(0) \zeta(5)}{64 \pi^{5}}-\frac{1}{120} U^{5}-\frac{i}{(2 \pi)^{5}} \sum_{(k, l)>0} c(k l) \mathcal{L} i_{5}\left[q_{T}{ }^{k} q_{U}{ }^{l}\right]+Q(T, U)
$$

with some quartic unconstrained polynomial $Q(T, U)$. The holomorphic covariant coupling $\partial_{T}^{5} \mathcal{G}$ is obtained by a five point amplitude $\left\langle V_{T} \prod_{i}^{4} V_{F_{T}}^{(i)}\right\rangle$ [10]. This is the analogue to the Yukawa couplings $\partial_{T}^{3} f$ in the $N=2$ supersymmetric case in $d=4$.

\subsection{Generalized worldsheet $\tau$-integrals}

Rather than restricting to the case (2.12), it is the purpose of this section to find general expressions for world-sheet $\tau$-integrals with an arbitrary number of lattice vector $\bar{p}_{R}, p_{R}, \bar{p}_{L}$ and $p_{L}$ insertions. Such integrals appear in $N$-point string amplitudes of toroidal string compactifications. See also [19] for further discussions and [19] [10] 20] 21] for examples.

$$
\begin{aligned}
\Delta_{q_{1} q_{2} q_{3} q_{4}} & :=\frac{\partial^{N}}{\partial \Lambda_{1}^{q_{1}} \partial \Lambda_{2}^{q_{2}} \partial \Lambda_{3}^{q_{3}} \partial \Lambda_{4}^{q_{4}}} \int \frac{d^{2} \tau}{\tau_{2}} \tau_{2}^{r} \frac{1}{\tau_{2}^{s}} \sum_{\left(p_{L}, p_{R}\right)} e^{\pi i \tau\left|p_{L}\right|^{2}} e^{-\pi i \bar{\tau}\left|p_{R}\right|^{2}} \\
& \times\left. e^{\Lambda_{1} \bar{p}_{R}+\Lambda_{2} p_{R}+\Lambda_{3} \bar{p}_{L}+\Lambda_{4} p_{L}} e^{-\frac{1}{2 \pi \tau_{2}}\left(\Lambda_{1} \Lambda_{2}+\Lambda_{2} \Lambda_{3}+\Lambda_{3} \Lambda_{4}+\Lambda_{4} \Lambda_{1}\right)} \bar{f}_{k}(\bar{\tau})\right|_{\Lambda_{i}=0}
\end{aligned}
$$

with the modular function $f_{k}$ of weight $k$, the integers $r, s \geq 0, q_{1}, q_{2}, q_{3}, q_{4} \geq 0$ and $N=q_{1}+q_{2}+q_{3}+q_{4}$. We exclude the trivial case $\left(q_{1}, q_{2}, q_{3}, q_{4}\right)=(0,0,0,0)$. World-sheet modular invariance of the integrand requires:

$$
\begin{aligned}
k & =-q_{1}-q_{2}+q_{3}+q_{4} \\
r-s-k & =q_{1}+q_{2} .
\end{aligned}
$$

5 The covariant derivative $D_{w}$ maps modular forms $\Phi_{w, \bar{w}}(U)$ of weight $(w, \bar{w})$ to forms of weight $(w+2, \bar{w})$ i.e. $\Phi_{w+2, \bar{w}}(U)=D_{w} \Phi_{w, \bar{w}}(U)=\frac{i}{\pi}\left(\partial_{U}+\frac{w}{(U-\bar{U})}\right) \Phi_{w, \bar{w}}(U)$. We use the notation $D^{k} \Phi_{w, \bar{w}}=D_{w+2(k-1)} D_{w+2(k-2)} \ldots D_{w} \Phi_{w, \bar{w}}$. 
In particular, the expression $(T-\bar{T})^{-m}(U-\bar{U})^{-n} \Delta_{q_{1} q_{2} q_{3} q_{4}}$ contains the piece

$$
\begin{aligned}
\mathcal{I}_{w_{T}, w_{U}, w_{\bar{T}}, w_{\bar{U}}} & =\frac{(T-\bar{T})^{-m}}{(U-\bar{U})^{n}} \\
& \times \int \frac{d^{2} \tau}{\tau_{2}^{1-r+s}} \sum_{\left(p_{L}, p_{R}\right)} \bar{p}_{R}^{q_{1}} p_{R}^{q_{2}} \bar{p}_{L}^{q_{3}} p_{L}^{q_{4}} e^{\pi i \tau\left|p_{L}\right|^{2}} e^{-\pi i \bar{\tau}\left|p_{R}\right|^{2}} \bar{f}_{k}(\bar{\tau})
\end{aligned}
$$

next to subleading pieces, which are less harmonic and are due to IR-effects originating from pinching vertex operators. In general, those terms have different modular weights. $\mathcal{I}_{w_{T}, w_{U}, w_{\bar{T}}, w_{\bar{U}}}$ has modular weights

$$
\begin{aligned}
& w_{T}=m+\frac{1}{2}\left(q_{1}-q_{2}-q_{3}+q_{4}\right) \\
& w_{\bar{T}}=m+\frac{1}{2}\left(q_{2}-q_{1}-q_{4}+q_{3}\right) \\
& w_{U}=n+\frac{1}{2}\left(q_{2}-q_{1}+q_{4}-q_{3}\right) \\
& w_{\bar{U}}=n+\frac{1}{2}\left(q_{1}-q_{2}+q_{3}-q_{4}\right)
\end{aligned}
$$

under $S L(2, \mathbf{Z})_{T} \times S L(2, \mathbf{Z})_{U},(a d-b c=1)$ :

$$
\begin{aligned}
& \left(p_{L}, \bar{p}_{R}\right) \rightarrow \sqrt{\frac{c T+d}{c \bar{T}+d}}\left(p_{L}, \bar{p}_{R}\right) \quad, \quad T \rightarrow \frac{a T+b}{c T+d}, U \rightarrow U, \\
& \left(p_{L}, p_{R}\right) \rightarrow \sqrt{\frac{c U+d}{c \bar{U}+d}}\left(p_{L}, p_{R}\right) \quad, \quad T \rightarrow T, U \rightarrow \frac{a U+b}{c U+d} .
\end{aligned}
$$

Poisson resummation on (2.15) leads to

$$
\begin{aligned}
\Delta_{q_{1} q_{2} q_{3} q_{4}} & :=T_{2} \frac{\partial^{N}}{\partial \Lambda_{1}^{q_{1}} \partial \Lambda_{2}^{q_{2}} \partial \Lambda_{3}^{q_{3}} \partial \Lambda_{4}^{q_{4}}} \int \frac{d^{2} \tau}{\tau_{2}^{2-r+s}} \sum_{A \in M(2, \mathbf{Z})} e^{-2 \pi i \bar{T} \operatorname{det} A} e^{\frac{-\pi T_{2}}{\tau_{2} U_{2}}\left|\left(\begin{array}{c}
1 \\
U
\end{array}\right)^{t} A\left(\begin{array}{c}
\tau \\
-1
\end{array}\right)\right|^{2}} \\
& \times\left. e^{-\frac{1}{\sqrt{2} \tau_{2}}\left(\Lambda_{1} \bar{Q}_{R}-\Lambda_{2} Q_{R}+\Lambda_{3} \bar{Q}_{L}-\Lambda_{4} Q_{L}\right)} \bar{f}_{k}(\bar{\tau})\right|_{\Lambda_{i}=0} \\
& =2^{-\frac{N}{2}} T_{2}(-1)^{q_{2}+q_{4}+N} \\
& \times \int \frac{d^{2} \tau}{\tau_{2}^{2+N-r+s}} \sum_{A \in M(2, \mathbf{Z})} \bar{Q}_{R}^{q_{1}} Q_{R}^{q_{2}} \bar{Q}_{L}^{q_{3}} Q_{L}^{q_{4}} e^{-2 \pi i \bar{T} \operatorname{det} A} e^{\frac{-\pi T_{2}}{\tau_{2} U_{2}}\left|\left(\begin{array}{c}
1 \\
U
\end{array}\right)^{t} A\left(\begin{array}{c}
\tau \\
-1
\end{array}\right)\right|^{2}} \bar{f}_{k}(\bar{\tau}) .
\end{aligned}
$$

Here the sum runs over all integer $2 \times 2$ matrices $A=\left(\begin{array}{c}n_{1}-l_{2} \\ n_{2} l_{1}\end{array}\right) \in M(2, \mathbf{Z})$. Modular invariance enables us to use the orbit decomposition used in [18], i.e. decomposing the set 
of all matrices $A$ into orbits of $S L(2, \mathbf{Z})$ :

$$
\begin{aligned}
& I_{0}: A=\left(\begin{array}{ll}
0 & 0 \\
0 & 0
\end{array}\right), \\
& I_{1}: A= \pm\left(\begin{array}{ll}
k & j \\
0 & p
\end{array}\right), 0 \leq j<k, p \neq 0, \\
& I_{2}: A=\left(\begin{array}{ll}
0 & j \\
0 & p
\end{array}\right), \quad(j, p) \neq(0,0) .
\end{aligned}
$$

Since we excluded the case $\left(q_{1}, q_{2}, q_{3}, q_{4}\right)=(0,0,0,0), I_{0}$ does not give any contribution. The integral to be done for the $I_{1}$ orbit is:

$$
\int_{0}^{\infty} \frac{d \tau_{2}}{\tau_{2}^{3 / 2}} e^{-a \tau_{2}-\frac{b}{\tau_{2}}}=\sqrt{\frac{\pi}{b}} e^{-2 \sqrt{a b}}
$$

After introducing

$$
\begin{aligned}
b & =p^{2}-\frac{i\left(\Lambda_{1}+\Lambda_{2}+\Lambda_{3}+\Lambda_{4}\right) p}{\pi \sqrt{2 T_{2} U_{2}}}-\frac{1}{8} \frac{\left(\Lambda_{1}-\Lambda_{2}+\Lambda_{3}-\Lambda_{4}\right)^{2}}{\pi^{2} T_{2} U_{2}} \\
\varphi & =p\left(k T_{1}+l U_{1}\right) \\
& +\frac{1}{2 \pi \sqrt{2 T_{2} U_{2}}}\left[\left(k T_{2}-l U_{2}\right) \Lambda_{1}+\left(-k T_{2}+l U_{2}\right) \Lambda_{2}+\left(-k T_{2}-l U_{2}\right) \Lambda_{3}+\left(k T_{2}+l U_{2}\right) \Lambda_{4}\right],
\end{aligned}
$$

and the functions

$$
\begin{aligned}
& I_{1}(\alpha, \beta)=\frac{2}{\sqrt{\beta b}} e^{-2 \pi\left(k T_{2}+l U_{2}\right) \sqrt{\alpha \beta b}} e^{-2 \pi i \varphi}, \\
& I_{2}(\alpha, \beta)=\frac{1}{\sqrt{\beta b}} e^{-2 \pi l U_{2} \sqrt{\alpha \beta b}}\left(e^{2 \pi i \varphi}+e^{-2 \pi i \varphi}\right) .
\end{aligned}
$$

we obtain the closed expressions for $I_{1}$ and $I_{2}$ in the chamber $T_{2}>U_{2}$ :

$$
\begin{aligned}
I_{1} & =\frac{\partial^{N}}{\partial \Lambda_{1}^{q_{1}} \partial \Lambda_{2}^{q_{2}} \partial \Lambda_{3}^{q_{3}} \partial \Lambda_{4}^{q_{4}}} \times \\
& \times\left.\sum_{\substack{k>0 \\
l \in \mathbf{Z}}} \sum_{p \neq 0} c(k l)\left[\frac{T_{2} U_{2}}{\pi\left(k T_{2}+l U_{2}\right)^{2}}\right]^{r} \frac{1}{\left(\pi T_{2} U_{2} b\right)^{s}} \frac{(-1)^{r+s} \partial^{r+s}}{\partial \alpha^{r} \partial \beta^{s}} I_{1}(\alpha, \beta)\right|_{\substack{\alpha=1 \\
\beta=1}, \Lambda_{i}=0},
\end{aligned}
$$




$$
\begin{aligned}
& I_{2}=\frac{\partial^{N}}{\partial \Lambda_{1}^{q_{1}} \partial \Lambda_{2}^{q_{2}} \partial \Lambda_{3}^{q_{3}} \partial \Lambda_{4}^{q_{4}}} \times \\
& \times\left.\sum_{\substack{k=0 \\
l>0}} \sum_{p \neq 0} c(0)\left[\frac{T_{2} U_{2}}{\pi\left(l U_{2}\right)^{2}}\right]^{r} \frac{1}{\left(\pi T_{2} U_{2} b\right)^{s}} \frac{(-1)^{r+s} \partial^{r+s}}{\partial \alpha^{r} \partial \beta^{s}} I_{2}(\alpha, \beta)\right|_{\substack{\alpha=1 \\
\beta=1}} \\
& +c(0) \operatorname{sgn}(s-r)^{s-r}\left(2 \pi T_{2} U_{2}\right)^{r-s}[1 \cdot 3 \cdot 5 \cdot \ldots \cdot(2|r-s|-1)]^{\operatorname{sgn}(s-r)} \sum_{p \neq 0} \frac{1}{b^{\frac{1}{2}+s-r}} \\
& +c(0) T_{2}^{r-s} \frac{U_{2}^{1+s-r}}{\pi^{1+s-r}}(-1)^{r+s} \frac{d^{s-r}}{d \xi^{s-r}} \sum_{j \neq 0} \frac{1}{j^{2}+j \frac{1}{\pi} \sqrt{\frac{U_{2}}{2 T_{2}}}\left(\Lambda_{2}+\Lambda_{4}-\Lambda_{1}-\Lambda_{3}\right)+\xi} \mid \begin{array}{c}
\xi=0 \\
\Lambda_{i}=0
\end{array} .
\end{aligned}
$$

Generically, the second sum leads to $\sum_{p>0} p^{-a}=\zeta(a)$ for $a=1+2(s-r)+N \neq 1$. For $a=1$ one has to replace this term with a regularized sum, which is derived in the appendix. In total, we obtain for (2.15):

$$
\Delta_{q_{1} q_{2} q_{3} q_{4}}=I_{1}+I_{2}^{r e g} .
$$

Let us briefly discuss the case $N:=q_{1} \neq 0$, even , $q_{2}, q_{3}, q_{4}=0, s=r=0$, for which $I_{1}, I_{2}$ can be further simplified:

$$
\begin{aligned}
& \Delta_{N}=\frac{\partial^{N}}{\partial \Lambda^{N}} \times \\
& \quad \times \sum_{\substack{k>0 \\
l \in \mathbf{Z}}} \sum_{p \neq 0} c(k l) \frac{2}{\left|p-\frac{i \Lambda}{2 \pi \sqrt{2 T_{2} U_{2}}}\right|} e^{-2 \pi\left(k T_{2}+l U_{2}\right)\left|p-\frac{i \Lambda}{2 \pi \sqrt{2 T_{2} U_{2}}}\right|} e^{-2 \pi i p\left(k T_{1}+l U_{1}\right)-\frac{i \Lambda}{\sqrt{2 T_{2} U_{2}}}\left(k T_{2}-l U_{2}\right)} \\
& \quad+\sum_{l>0} c(0) \sum_{p \neq 0} e^{-2 \pi l U_{2}\left|p-\frac{i \Lambda}{2 \pi \sqrt{2 T_{2} U_{2}}}\right|}\left(e^{2 \pi i l p U_{1}} e^{-\Lambda i l \sqrt{\frac{U_{2}}{2 T_{2}}}}+e^{-2 \pi i l p U_{1}} e^{\Lambda i l \sqrt{\frac{U_{2}}{2 T_{2}}}}\right) \\
& \quad+2 c(0) \zeta(N+1) N !\left(\frac{i}{2 \pi \sqrt{2 T_{2} U_{2}}}\right)^{N}+2^{\frac{1}{2} N+\left.2 c(0) U_{2}\left(\frac{U_{2}}{T_{2}}\right)^{\frac{N}{2}} \frac{\pi}{(N+1)(N+2)} B_{\frac{N}{2}+1}\right|_{\Lambda=0} .}
\end{aligned}
$$

Now let us come back to eq. (2.12). Unfolding the integration (2.21) enables one to identify these contributions to tree-level, higher perturbative and non-perturbative corrections on the type I side. Let us briefly discuss a case, for which $I_{1}, I_{2}$ can be simplified further: $q_{1}, q_{2} \neq 0 ; v, w=0, s=r=0$ with $q \equiv q_{2}$. The non-degenrate orbit gives:

$$
\Delta_{F_{1}^{4-q} F_{2}^{q}}^{\text {non-deg }}=\frac{\partial^{4-q}}{\partial \Lambda_{1}^{4-q}} \frac{\partial^{q}}{\partial \Lambda_{2}^{q}} \sum_{\substack{0 \leq j<k \\ p \neq 0}} \frac{1}{k \sqrt{b\left(\Lambda_{1}, \Lambda_{2}\right)}} e^{-2 \pi i k p \tilde{T}\left(\Lambda_{1}, \Lambda_{2}\right)} \mathcal{A}\left[\tilde{U}\left(\Lambda_{1}, \Lambda_{2}\right)\right],
$$

where

$$
\begin{aligned}
& \tilde{T}\left(\Lambda_{1}, \Lambda_{2}\right)=T_{1}-\frac{i}{p} T_{2}\left[\sqrt{b\left(\Lambda_{1}, \Lambda_{2}\right)}+\frac{i\left(\Lambda_{1}-\Lambda_{2}\right)}{2 \pi \sqrt{2 T_{2} U_{2}}}\right] \\
& \tilde{U}\left(\Lambda_{1}, \Lambda_{2}\right)=\frac{1}{k}\left(j+p U_{1}-i U_{2}\left[\sqrt{b\left(\Lambda_{1}, \Lambda_{2}\right)}+\frac{i\left(\Lambda_{1}-\Lambda_{2}\right)}{2 \pi \sqrt{2 T_{2} U_{2}}}\right]\right) .
\end{aligned}
$$


The worldsheet instanton corrections $\Delta^{\text {non-deg }}$ are exponentially suppressed. From the point of view of heterotic-type I duality it is convenient to rewrite this expression in terms of Hecke operators [7]:6]

$$
\begin{aligned}
\Delta_{F_{1}^{4-q} F_{2}^{q}}^{\text {non-deg }} & =\frac{\pi^{4}}{2} \sum_{N=1}^{\infty}\left[\left(\frac{T_{2}}{U_{2}}\right)^{2-q}\left(D_{\mathcal{T}}^{4-q} q_{\mathcal{T}}\right) N^{4-q} H_{N}\left[D_{U}^{q} \mathcal{A}_{-4}\right](U)\right. \\
& \left.+\left(\frac{U_{2}}{T_{2}}\right)^{2-q} D_{\mathcal{T}}^{q} \bar{q}_{\overline{\mathcal{T}}} \frac{1}{N^{4-q}} H_{N}\left[\bar{D}_{\bar{U}}^{4-q} \overline{\mathcal{A}}_{-4}\right](\bar{U})\right]
\end{aligned}
$$

where $q_{\mathcal{T}}=e^{2 \pi i \mathcal{T}}$ with $\mathcal{T}=N T$ and covariant derivatives acting on $q_{\mathcal{T}}$ start with $D_{-4}^{\mathcal{T}}$. The structure of covariant derivatives in the above expression matches with the one found in $(2.13)$.

The degenerate orbit gives

$$
\Delta_{F_{1}^{4-q} F_{2}^{q}}^{\mathrm{deg}}=\frac{c_{0}}{\pi} \Gamma(5) \frac{U_{2}^{3}}{T_{2}^{3}} \sum_{(j, p) \neq(0,0)} \frac{(j-p U)^{4-q}(j-p \bar{U})^{q}}{|j-p U|^{10}}
$$

where $c_{0} \equiv c(0)=504$ is the constant coefficient of the light-cone Ramond partition function $\frac{\bar{E}_{4}^{2}}{\bar{\eta}^{24}}=\sum_{n} c(n) \bar{q}^{n}$. The sum is taken over winding modes $j, p$. The case $n_{1}=$ $n_{2}=0$ in (2.11) corresponds to vanishing winding numbers. Only Kaluza-Klein momenta contribute to $\Delta^{\text {deg}}$, which will be identified with type I perturbative corrections in the next section. For $q=2$ the result can be expressed in terms of the generalized Eisenstein function $E(U, 3)=U_{2}^{3} \zeta(6) \sum_{(j . p) \neq 0} \frac{1}{|j-p U|^{6}}$ [23].

\section{4. $R^{4} T^{4 g-4}$ couplings}

The heterotic effective action compacified on $K 3 \times T^{2}$ contains higher derivative couplings $F_{g} \operatorname{Tr} R^{2} T^{2 g-2}$, where $T_{\mu \nu}$ is the field strength of the graviphoton. These couplings appear at one loop [21] and only depend on the vector multiplet scalars. They do not depend on the $K 3$ moduli. T Therefore the moduli-dependence of these couplings should survive in the limit of large $K 3$, i.e. in the decompactification to $d=8$. In fact, we will show in this section, that the one-loop corrections to the couplings $F_{g} \operatorname{Tr} R^{4} T^{4 g-4}$ and $F_{g}\left(\operatorname{Tr} R^{2}\right)^{2} T^{4 g-4}$ share the same moduli dependence. They are derived from a one-loop

6 The Hecke operator acts on a modular form $\Phi_{w}$ of weight $w$ as $H_{N}\left[\Phi_{w}\right](U)=$ $\frac{1}{N^{1-w}} \sum_{\substack{k, p>0 \\ k p=N}} \sum_{0 \leq j<k} k^{-w} \Phi_{w}(\mathcal{U})$ with the $D$-brane complex structure $\mathcal{U}=\frac{j+p U}{k}$ [22].

7 However, they depend on the chosen gauge bundle. See e.g.: [24]. 
amplitude with four graviton and $(4 g-4)$ graviphoton vertex operators. Since the $C P$ odd amplitude is related to the $C P$ even amplitude by supersymmetry, which results in the same coupling, it is sufficient to discuss the $C P$ even case only. This part is highly constrained by $U(1)$ charge conservation of the internal $c=3$ conformal field theory. Essentially this means, that only the bosonic part of the graviphoton vertex operators gives non-vanishing contributions. For the $C P$-even amplitude we use (2.1) as graviton vertex operator in zero ghost picture and the following graviphoton vertex operator in background gauge:

$$
V_{g p h}^{(0)}=-\frac{1}{2} T_{\mu \nu} \bar{\partial} X^{\mu}\left[X^{\nu} \epsilon_{I} \partial X^{I}-\frac{1}{4} \epsilon_{I} \gamma_{a b}^{\nu I} S^{a} S^{b}\right]
$$

The eight fermionic zero modes are soaked up by the graviton vertex operator and we get for the amplitude

$$
\begin{aligned}
\mathcal{A} & =\left\langle\int \prod_{i=1}^{4} d^{2} z_{i} V_{g, i}^{(0)}\left(z_{i}\right) \int \prod_{j=1}^{4 g-4} d^{2} z_{j} V_{g p h, j}^{(0)}\left(z_{j}\right)\right\rangle \\
& =\left(\frac{1}{2}\right)^{4 g} t^{\nu_{1} \sigma_{1} \nu_{2} \sigma_{2} \nu_{3} \sigma_{3} \nu_{4} \sigma_{4}} \prod_{i=1}^{4} R_{\mu_{i} \rho_{i} \nu_{i} \sigma_{i}} \prod_{i=5}^{4 g} T_{\mu_{i} \rho_{i}} \\
& \times \int \frac{d^{2} \tau}{\tau_{2}^{5}} \frac{\bar{E}_{4}^{2}}{\bar{\eta}^{24}} \sum_{p_{L}, p_{R}}\left(\frac{p_{L}}{\sqrt{2 T_{2} U_{2}}}\right)^{4 g-4} q^{\frac{1}{2} p_{L}^{2}} \bar{q}^{\frac{1}{2} p_{R}^{2}}\left\langle\int \prod_{i=1}^{4 g} d^{2} z_{i} \bar{\partial} X^{\mu_{i}}\left(z_{i}\right) X^{\rho_{i}}\left(z_{i}\right)\right\rangle .
\end{aligned}
$$

For the bosonic correlation function we introduce the generating functional

$$
G\left(\tilde{\lambda}_{1}, \tilde{\lambda}_{2}, T, U\right)=\sum_{g=1} \frac{1}{2 g !^{2}}\left(\frac{\tilde{\lambda}_{1} \tilde{\lambda}_{2}}{\tau_{2}}\right)^{2 g-2}\left\langle\prod_{i=1}^{2 g} \bar{\partial} X^{\mu_{i}} X^{\nu_{i}}\right\rangle^{2}=\tau_{2}^{4} \frac{d^{4}}{d \tilde{\lambda}_{1}^{2} d \tilde{\lambda}_{2}^{2}}\left\langle e^{-S_{0}+\tilde{\lambda}_{1} S}\right\rangle\left\langle e^{-S_{0}+\tilde{\lambda}_{2} S}\right\rangle
$$

with $\tilde{\lambda}_{i}=\frac{\lambda_{i} \tau_{2} p_{L}}{\sqrt{2 T_{2} U_{2}}}$ and $8 S_{0}-\tilde{\lambda} S=\frac{1}{\pi} \int d^{2} z\left(\partial X^{\mu} \bar{\partial} X_{\mu}-\frac{\tilde{\lambda}}{\tau_{2}} X^{\mu} \bar{\partial} X_{\mu}\right)$ and

$$
\left\langle e^{-S_{0}+\lambda S}\right\rangle=\frac{|\eta|^{4}}{\left(\operatorname{det}^{\prime} \Delta\right)^{2}}=\left[\frac{\lambda \theta_{1}^{\prime}(0, \bar{\tau})}{\theta_{1}(\tilde{\lambda}, \bar{\tau})}\right]^{2} e^{\frac{-\pi \tilde{\lambda}^{2}}{\tau_{2}}}
$$

where $\left(\operatorname{det}^{\prime} \Delta\right)^{d / 2}=|\eta|^{d}\left[\frac{\theta_{1}(\tilde{\lambda}, \bar{\tau})}{\bar{\eta}^{3} \tilde{\lambda} 2 \pi}\right]^{d / 2} e^{\frac{d \pi \tilde{\lambda}^{2}}{4 \tau_{2}}}$ is the determinant for $d$ scalar fields on a worldsheet torus which is calculated using $\zeta$-function regularization [21]. We introduced the

8 In analogy to 21] we evaluate the determinant by passing to a complex basis for the eight dimensional Euclidean space: $Z^{1}=\frac{1}{\sqrt{2}}\left(X^{1}-i X^{2}\right), Z^{2}=\frac{1}{\sqrt{2}}\left(X^{0}-i X^{3}\right), Z^{3}=\frac{1}{\sqrt{2}}\left(X^{5}-i X^{6}\right)$, $Z^{4}=\frac{1}{\sqrt{2}}\left(X^{4}-i X^{7}\right)$. 
dependence on $\tilde{\lambda}_{1}$ and $\tilde{\lambda}_{2}$ which keeps track of the space-time kinematics of the tensors $R_{\mu \nu}$ and $T_{\mu \nu}$ w.r.t. complex bosons $Z^{1}, Z^{2}$ and $Z^{3}, Z^{4}$, respectively. We may summarize all couplings $F_{g}$ by the generating function

$$
\begin{aligned}
F\left(\tilde{\lambda}_{1}, \tilde{\lambda}_{2}, T, U\right) & =\sum_{g} F_{g}\left(\lambda_{1} \lambda_{2}\right)^{2 g-2}=\int \frac{d^{2} \tau}{\tau_{2}} \frac{\bar{E}_{4}^{2}}{\bar{\eta}^{24}} \sum_{p_{L}, p_{R}} q^{\frac{1}{2} p_{L}^{2}} \bar{q}^{\frac{1}{2} p_{R}^{2}} \\
& \times \frac{d^{4}}{d \tilde{\lambda}_{1}^{2} d \tilde{\lambda}_{2}^{2}}\left[\frac{2 \pi i \tilde{\lambda}_{1} \bar{\eta}^{3}}{\theta_{1}\left(\tilde{\lambda}_{1}, \bar{\tau}\right)} e^{-\frac{\pi \tilde{\lambda}_{1}^{2}}{2 \tau_{2}}} \frac{2 \pi i \tilde{\lambda}_{2} \bar{\eta}^{3}}{\theta_{1}\left(\tilde{\lambda}_{2}, \bar{\tau}\right)} e^{-\frac{\pi \tilde{\lambda}_{2}^{2}}{2 \tau_{2}}}\right]^{2} .
\end{aligned}
$$

Using the identity $\frac{\tilde{\lambda} \theta_{1}^{\prime}}{\theta_{1}(\tilde{\lambda}, \bar{\tau})} e^{-\frac{\pi \tilde{\lambda}^{2}}{2 \tau_{2}}}=\exp \left[\sum_{k} \frac{1}{2 k} \tilde{\lambda}^{2 k} \hat{G}_{2 k}\right]$ the above expression can be compared with the gravitational part of the elliptic genus (2.4), replacing $\frac{R}{4 \pi^{2}}$ by $\tilde{\lambda}$.

We use a somewhat different way than the one in 20] to integrate (2.37). In fact, similar as in [11], it proves to be useful to promote the Jacobi-form $\Phi_{-2}(\tau, \mathbf{z})=\frac{1}{\theta_{1}\left(z_{1}, \tau\right)^{2} \theta_{1}\left(z_{2}, \tau\right)^{2}}$ of characteristics $(w, m)=(-2,-1)$ to the almost holomorphic function

$$
\tilde{\Phi}_{-2}(\tau, \mathbf{z})=\frac{1}{\theta_{1}\left(z_{1}, \tau\right)^{2} \theta_{1}\left(z_{2}, \tau\right)^{2}} e^{\pi \frac{\mathbf{z}^{2}}{\tau_{2}} m} \quad, \quad m=-1
$$

with the well-behaved modular behaviour $\tilde{\Phi}_{-2}\left(\frac{a \tau+b}{c \tau+d}, \frac{\mathbf{z}}{c \tau+d}\right)=(c \tau+d)^{-2} \tilde{\Phi}_{-2}(\tau, \mathbf{z})$. Thus all the non-holomorphic parts of the genus are captured by $\tilde{\Phi}_{-2}$. We treat the $p_{L}^{4 g-4}$-lattice vector insertions as before in eq. 2.15) and obtain for $F_{g}$ the closed form

$$
\begin{aligned}
& F_{g}=\frac{1}{(2 g) !^{2}} \frac{1}{\left(2 T_{2} U_{2}\right)^{2 g-2}} \frac{d^{4 g-4}}{d \Lambda^{4 g-4}} \frac{d^{4 g}}{d \bar{z}_{1}^{2 g} d \bar{z}_{2}^{2 g}} \times
\end{aligned}
$$

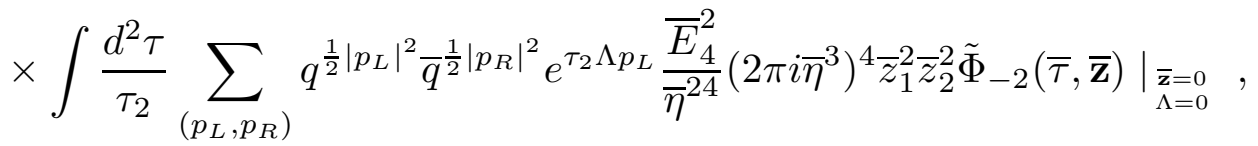

in which the $\tau$-integration becomes straightforward. We need the coefficients $c(n ; \mathbf{b})$ appearing in the power series? $\left(y_{k}=e^{2 \pi i z_{k}}\right)$ :

$$
\frac{E_{4}^{2}}{\eta^{24}}\left(2 \pi i \eta^{3}\right)^{4} z_{1}^{2} z_{2}^{2} \Phi_{-2}(\tau, z)=\frac{E_{4}^{2}}{\eta^{24}} \sum_{b_{1}, b_{2}=0}^{\infty} z_{1}^{2 b_{1}} z_{2}^{2 b_{2}} \mathcal{P}_{2 b_{1}} \mathcal{P}_{2 b_{2}}=\sum_{b_{1}, b_{2}=0}^{\infty} \sum_{n \geq-1} c(n ; \mathbf{b}) q^{n} z_{1}^{2 b_{1}} z_{2}^{2 b_{2}}
$$

Only $F_{1}$ receives a contribution from the trivial orbit of (2.21), namely

$$
I_{0}(g)= \begin{cases}\frac{16}{3} \pi^{5} T_{2} & : \quad g=1 \\ 0: & g \neq 1\end{cases}
$$

9 The functions $\mathcal{P}_{2 b}\left(G_{2}, \ldots, G_{2 b}\right)$ have been introduced in [20]. E.g.: $\mathcal{P}_{0}=-1, \quad \mathcal{P}_{2}=$ $-G_{2}, \mathcal{P}_{4}=-\frac{1}{2}\left(G_{2}^{2}+G_{4}\right), \mathcal{P}_{6}=-\frac{1}{3} G_{6}-\frac{1}{2} G_{2} G_{4}-\frac{1}{6} G_{2}^{3}, \mathcal{P}_{8}=-\frac{3}{8} G_{4}^{2}-\frac{1}{3} G_{2} G_{6}-\frac{1}{4} G_{4} G_{2}^{2}-\frac{1}{24} G_{2}^{4}$. 
Proceeding with the orbits (2.21) we obtain for the non-degenerate orbit 10 (in the chamber $\left.T_{2}>U_{2}\right)$ :

$$
\begin{aligned}
I_{1}(g) & =\frac{1}{(2 g) !^{2}} \frac{1}{\left(2 T_{2} U_{2}\right)^{2 g-2}} \frac{d^{4 g-4}}{d \Lambda^{4 g-4}} \frac{d^{4 g}}{d z_{1}^{2 g} d z_{2}^{2 g}} \sum_{\mathbf{b}} \sum_{(k, l)>0} c(k l ; \mathbf{b}) z_{1}^{2 b_{1}} z_{2}^{2 b_{2}} \\
& \times\left.\sum_{p \neq 0} \frac{2}{\sqrt{p^{2}-\frac{m \mathbf{z}^{2}}{T_{2} U_{2}}}} e^{-2 \pi \sqrt{p^{2}-\frac{m \mathbf{z}^{2}}{T_{2} U_{2}}}\left|k T_{2}+l U_{2}+\frac{i \Lambda \sqrt{T_{2} U_{2}}}{2 \pi \sqrt{2}}\right|} e^{-2 \pi i p\left[\left(k T_{1}+l U_{1}\right)-\frac{\Lambda \sqrt{T_{2} U_{2}}}{2 \pi \sqrt{2}}\right]}\right|_{\substack{\Lambda=0 \\
\mathbf{z}=0}} \\
& =\frac{1}{(2 g) !^{2}} \frac{(-1)^{2 g-2}}{2^{4 g-5}} \frac{d^{4 g}}{d z_{1}^{2 g} d z_{2}^{2 g}} \sum_{b} \sum_{(k, l)>0} c(k l ; \mathbf{b}) z_{1}^{2 b_{1}} z_{2}^{2 b_{2}} \\
& \times\left.\sum_{p \neq 0} \frac{\left(p-\sqrt{p^{2}-\frac{m \mathbf{z}^{2}}{T_{2} U_{2}}}\right)^{4 g-4}}{\sqrt{p^{2}-\frac{m \mathbf{z}^{2}}{T_{2} U_{2}}}} e^{-2 \pi \sqrt{p^{2}-\frac{m \mathbf{z}^{2}}{T_{2} U_{2}}}\left(k T_{2}+l U_{2}\right)} e^{-2 \pi i p\left(k T_{1}+l U_{1}\right)}\right|_{\mathbf{z}=0} .
\end{aligned}
$$

We have checked, that after some work, (2.42) can be written for $z_{1}=z_{2}$ in the form eq. (4.40) of [20]. In (2.42), the sum $p<0$ gives rise to $T, U$ dependent polylogarithmic contributions, whereas $p>0$ leads to $\bar{T}, \bar{U}$ dependent pieces, which vanish for $g \geq 3$. For the degnerate orbit we find:

$$
\begin{aligned}
I_{2}(g) & =\frac{1}{(2 g) !^{2}} \frac{d^{4 g}}{d z_{1}^{2 g} d z_{2}^{2 g}} \sum_{\mathbf{b}} 2^{4-4 g} c(0 ; \mathbf{b}) z_{1}^{2 b_{1}} z_{2}^{2 b_{2}} \\
& \times\left.\left\{(-1)^{2 g-2} \sum_{p \neq 0} \frac{\left(p-\sqrt{p^{2}-\frac{m \mathbf{z}^{2}}{T_{2} U_{2}}}\right)^{4 g-4}}{\sqrt{p^{2}-\frac{m \mathbf{z}^{2}}{T_{2} U_{2}}}}+\frac{2 U_{2}^{5-4 g}}{\pi} \sum_{j=1}^{\infty} \frac{j^{4 g-4}}{j^{2}-m \mathbf{z}^{2} \frac{U_{2}}{T_{2}}}\right\}\right|_{z=0} .
\end{aligned}
$$

For $g=1$ the first sum in the bracket of (2.43) is divergent if none of the $z_{i}$-derivatives act on it. In general, this divergence occurs when $(4 g-4) z_{i}$-derivatives act on the sum over $p$. In these cases the sum has to be replaced by a regularized expression, which we present in the appendix. With that in mind, we get in total for (2.39):

$$
F_{g}=I_{0}(g)+I_{1}(g)+I_{2}^{r e g} \cdot(g)
$$

We want to extract the holomorphic piece of $F_{g}$, which has a topological interpretation on the dual IIB side, described by $F$-theory on $K 3$. The latter is described by an $\mathrm{N}=4$ superconformal field theory and $4 g-4$ graviphoton insertions lead to topological invariant correlators at $g$-loop order [25]. The holomorphic piece $F_{g}^{\text {holom. }}$ appears from (2.42) and (2.43)

10 We already include the $k=0, l>0$ contribution coming from the degenerate orbit $I_{2}(g)$. 
in the limit $\bar{T}, \bar{U} \rightarrow i \infty$, i.e. when all $z$-derivatives act on the genus $\sum_{\mathbf{b}} c(n ; \mathbf{b}) z_{1}^{2 b_{1}} z_{2}^{2 b_{2}}$ :

$$
F_{g \geq 2}^{\text {holom }} \cdot(T, U)=\zeta(5-4 g) c(0 ; 2 g, 2 g)+2 \sum_{(k, l)>0} c(k l ; 2 g, 2 g) \mathcal{L} i_{5-4 g}\left[q_{T}{ }^{k} q_{U}{ }^{l}\right]
$$

The harmonic part of $F_{1}$ is given by:

$$
F_{1}^{h a r m}(T, U, \bar{T}, \bar{U})=-\frac{24 i \pi^{5}}{9} T-\frac{76 i \pi^{5}}{9} U+2 \sum_{(k, l)>0} c(k l ; 2,2) \mathcal{L} i_{1}\left[q_{T}^{k} q_{U}^{l}\right]+h c
$$

whereas the case $g=0$ is related to (2.14). After (2.40), the coefficients $c(k l ; 2 g, 2 g)$ refer to $\mathcal{P}_{0}^{2} E_{4}^{2} \eta^{-24}=E_{4}^{2} \eta^{-24}, \mathcal{P}_{2}^{2} E_{4}^{2} \eta^{-24}=G_{2}^{2} E_{4}^{2} \eta^{-24}$ and $\mathcal{P}_{4}^{2} E_{4}^{2} \eta^{-24}=\frac{1}{4}\left(G_{4}+G_{2}^{2}\right)^{2} E_{4}^{2} \eta^{-24}$ for $g=0,1$ and $g=2$, respectively. Clearly, for $g=0$ we just get the holomorphic prepotential $\mathcal{G}$ (2.14), which counts genus 0 curves of the underlying $K 3$ [10]. The $g=1$ case gives the correction to $\left(\operatorname{tr} R^{2}\right)^{2}$, whereas the $g=2$ case corresponds to $\left(\operatorname{tr} R^{2}\right)^{2} T^{4}$. Different distributions of $z_{1}, z_{2}$-derivatives in (2.39) lead to different Lorentz kinematics. In particular it is possible to find a combination which gives a relation

$$
F_{g}^{\text {holom }} \leftrightarrow\left(\frac{d}{d q} G_{2}(q)\right)^{g} \frac{q}{\eta(q)^{24}} E_{4}(q)^{2}
$$

to the counting formula [26]. The latter counts genus $g$ curves with $g$ nodes on the $K 3$ of the dual $F$-theory compactification. A similar correspondence has been recently established in four dimensions in counting curves of the $K 3$ fiber of a Calabi-Yau manifold [20].

The amplitudes $F_{g}$ have no obvious relation to the $R^{4} H^{4 g-4}$ IIB couplings of [27]. In particular, the latter contain the set of $(p, q) D$-string contributions, which does not exist in our vacua. Moreover the couplings (2.45) behave like $F_{g} \rightarrow \zeta(5-4 g) c(0 ; 2 g, 2 g)$ in the decompactification limit $T \rightarrow i \infty$ to ten dimensions in contrast to the IIB coupings $R^{4} H^{4 g-4}$, which give the famous $D$-instanton sum.

\section{Type $I$ in eight dimensions}

In this section we discuss two type I models $(A)$ and $(B)$. Model $(A)$ has $E_{8} \times$ $E_{8} \times U(1)^{2}$ and model $(B) S O(8)^{4} \times U(1)^{2}$ gauge group. For model $(A)$ we calculate gauge threshold corrections w.r.t. to the $U(1)$ factors, while for model $(B)$ we derive the corrections w.r.t. to the $S O(8)$. Furthermore, we discuss type I $R^{2}$ corrections. 


\section{1. $F^{4}$ type I couplings in model $(A)$}

The dilaton dependence for surfaces with Euler number $\chi=2-2 g-B-C$, is $\lambda_{I}^{-\chi}$ where $g$ is the genus, $B$ and $C$ the number of boundaries and crosscaps, respectively. Using the duality map (1.1) we verify that the degenerate orbit of the heterotic threshold corrections 2.32

$$
S_{\text {deg }}^{\text {het }}=\frac{V^{(8)} T_{2}}{2^{8} \pi^{4}} t_{8} F_{1}^{4-q} F_{2}^{q} \Delta_{F_{1}^{4-q} F_{2}^{q}}^{\text {deg }}\left(T_{2}, U\right) \leftrightarrow S_{1-\text { loop }}^{\mathrm{I}},
$$

appear at one loop in the type I effective action. The term $V^{(8)} t_{8}$ is invariant under this transformation, and the factor $e^{2 \Phi^{I}}$ which arises from $\frac{T_{2}}{T_{2}^{3}}$ is canceled by $G_{\mathrm{int}}^{-2}$ contracting the internal indices of the gauge kinetic term. Thus on the type I side we get the corresponding one loop $\left(\lambda_{I}^{0}\right)$ coupling.

Now let us directly compute (3.1) in type I string theory. One loop open string amplitudes consist of summing over oriented and unoriented surfaces with and without boundaries like the torus $(\mathcal{T})$ and Klein bottle $(\mathcal{K})$ for the closed string sector and the annulus $(\mathcal{A})$ and Möbius strip $(\mathcal{M})$ for the open string sector, which have Euler number $\chi=0$.

The vertex operator for Abelian gauge fields coincides with the one of type IIB theory:

$$
V_{\text {gauge }}=G_{\mu I}:\left(\bar{\partial} X^{I}-\frac{1}{4} k_{\sigma} \bar{S}^{a} \gamma_{a, b}^{I \sigma} \bar{S}^{b}\right)\left(\partial X^{\mu}-\frac{1}{4} k_{\nu} S^{a} \gamma_{a b}^{\nu \mu} S^{b}\right) e^{i k X}:
$$

There is no contribution from the torus diagram $\mathcal{T}$ since the sixteen fermionic zero modes cannot be saturated at the level of four derivative terms. The remaining amplitudes can be written as:

$$
\begin{aligned}
S_{1-\text { loop }}^{I} & =\frac{1}{2} V^{(8)} \sum_{\sigma=\mathcal{K}, \mathcal{A}, \mathcal{M}} \rho_{\sigma} \int_{0}^{\infty} \frac{d t}{t} \frac{1}{\left(2 \pi^{2} t\right)^{4}}\left(\sum_{p \in \Gamma_{2}} e^{-\pi t|p|^{2} / 2}\right) Z\left(\tau_{\sigma}\right) \\
& \times \int \prod_{i=1}^{4} d^{2} z_{i}\left\langle\prod_{i=1}^{4} V_{\text {gauge }, i}\right\rangle
\end{aligned}
$$

with relative weights $\rho_{\mathcal{K}}=1, \rho_{\mathcal{A}}=N^{2}$ and $\rho_{\mathcal{M}}=-N$, and $N$ is the Chan-Paton charge which is $N=32$ for $S O(32)$. The factor $\left(2 \pi^{2} t\right)^{-4}$ arises from momentum integration and $V^{(8)}$ is the uncompactified volume in type I units. The open string oscillator sum is $Z\left(\tau_{\sigma}\right)=\frac{1}{\eta^{12}\left(\tau_{\sigma}\right)} \sum_{\alpha=2,3,4} \frac{1}{2} s_{\alpha} \theta_{\alpha}^{4}\left(0, \tau_{\sigma}\right)$ with GSO projection signs $s_{3}=-s_{2}=-s_{4}=1$ and modular parameters $\tau_{\mathcal{A}}=\frac{i t}{2}, \tau_{\mathcal{M}}=\frac{i t+1}{2}, \tau_{\mathcal{K}}=2 i t$. The perturbative duality group for the 
open string is reduced to $S L(2, \mathbf{Z})_{U}$ and the torus partition function $\Gamma_{2}$ is now restricted to the Kaluza-Klein momenta $m_{1}$ and $m_{2}$ and $p^{2}=p_{I} G^{I J} p_{J}=\frac{1}{2 U_{2} \sqrt{G}}\left|m_{1}+m_{2} U\right|^{2}$.

Contraction of the leftmoving fermions $S^{a} \gamma_{a b}^{\nu \mu} S^{b}$ contributes four derivatives to the amplitude and using a Riemann identity resulting in

$$
Z(t) G_{F}^{4}(t)=-\frac{\pi^{4}}{2}
$$

one finds after Poisson resummation:

$$
\begin{aligned}
S_{1-\text { loop }}^{I} & =\frac{V^{(8)} T_{2}}{2^{8}} t_{8} F_{1}^{4-q} F_{2}^{q} \int \frac{d t}{t^{6}} \sum_{j, p \neq 0} e^{\frac{-\pi T_{2}}{t U_{2}}|j-p U|^{2}} \frac{T_{2}^{2}}{U_{2}^{2}} \\
& \times(j-p U)^{4-q}(j-p \bar{U})^{q} \frac{1}{2}\left(N^{2}-N+2^{4}\right)
\end{aligned}
$$

where $\frac{1}{2}\left(N^{2}-N+2^{4}\right)=c_{0}$. Integration over $t$ thus reproduces the corresponding heterotic coupling $\Delta_{F_{1}^{4-q} F_{2}^{q}}^{\text {deg }}\left(T_{2}, U\right)$.

\section{2. $R^{4} T^{4 g-4}$ type I couplings}

The relevant type I one-loop amplitude involves four gravitons and $(4 g-4)$ graviphotons whose vertex operators are:

$$
\begin{gathered}
V_{\text {grav }}=\epsilon_{\mu \nu}:\left(\bar{\partial} X^{\nu}-\frac{1}{4} \bar{S}^{a} \gamma_{a b}^{\nu \rho} \bar{S}^{b} k_{\rho}\right)\left(\partial X^{\mu}-\frac{1}{4} S^{a} \gamma^{\mu \sigma} S^{b} k_{\sigma}\right) e^{i k X}: \\
V_{\text {gph }}=G_{\mu I}:\left(\bar{\partial} X^{\mu}-\frac{1}{4} k_{\sigma} \bar{S}^{a} \gamma_{a, b}^{\mu \sigma} \bar{S}^{b}\right)\left(\partial X^{I}-\frac{1}{4} k_{\nu} S^{a} \gamma_{a b}^{\nu I} S^{b}\right) e^{i k X}:
\end{gathered}
$$

In analogy to 28] we can immediately write down the expression for the generating functional:

$$
F_{g}^{I}(\lambda)=\sum_{\sigma=\mathcal{T}, \mathcal{A}, \mathcal{M}, \mathcal{K}} \rho_{\sigma} \int_{0}^{\infty} \frac{d t}{t} \sum_{p \in \Gamma_{2}} e^{-\pi t|p|^{2} / 2} \frac{d^{4}}{d \tilde{\lambda}^{4}}\left\langle e^{S_{0}+\tilde{\lambda} S_{\mathrm{inst}}}\right\rangle_{\sigma}
$$

with $\left\langle e^{S_{0}+\tilde{\lambda} S_{\text {inst }}}\right\rangle_{\mathcal{A}}=\left\langle e^{S_{0}+\tilde{\lambda} S_{\text {inst }}}\right\rangle_{\mathcal{M}}=\left(\frac{\pi \tilde{\lambda}}{\sin \pi \tilde{\lambda}}\right)^{4}$ and $\left\langle e^{S_{0}+\tilde{\lambda} S_{\text {inst }}}\right\rangle_{\mathcal{K}}=\left(\frac{2 \pi \tilde{\lambda}}{\tan \pi \tilde{\lambda}}\right)^{4}$. The torus amplitude only contributes to $F_{1}^{I}$, which yields $\ln U_{2}|\eta(U)|^{4}$ [29] from summing up eveneven and odd-odd spin structures.

The final expression is:

$$
\begin{aligned}
F_{g}^{I}(\lambda) & =\int_{0}^{\infty} \frac{d t}{t} \sum_{p \in \Gamma_{2}} e^{-\pi t|p|^{2} / 2}\left[\frac{N^{2}-N+2^{4}}{2} \frac{d^{4}}{d \tilde{\lambda}^{4}}\left(\frac{\pi \tilde{\lambda}}{\sin \pi \tilde{\lambda}}\right)^{4}\right. \\
& \left.+\frac{d^{4}}{d \tilde{\lambda}^{4}}\left((2 \pi \tilde{\lambda})^{4}-2 \frac{(2 \pi \tilde{\lambda})^{4}}{(\sin \pi \tilde{\lambda})^{2}}\right)\right]+F_{1}^{\text {torus }}
\end{aligned}
$$

which can be identified with the degenrate orbit of (2.37) for $\tilde{\lambda}_{1}=\tilde{\lambda}_{2}=\lambda$. 


\section{3. $S O(8)^{4}$ type I threshold corrections in model $(B)$}

In this section we discuss the orientifold example of Sen [30]. For this model heterotic/F-theory duality can be checked explicitly [10]. The (discrete) Wilson lines

$a_{1}^{I}=\frac{1}{2}\left(0^{4}, 0^{4}, 1^{4}, 1^{4}\right)$ and $a_{2}^{I}=\frac{1}{2}\left(0^{4}, 1^{4}, 0^{4}, 1^{4}\right)$ break the gauge group to $S O(8)^{4}$. For this model the internal Abelian gauge group cannot be enhanced [2] and the underlying prepotenial is trivial. I.e. in this case the corresponding one-loop gauge couplings $\Delta_{F_{1}^{4-q} F_{2}^{q}}$ in (2.13) vanish identically [10].

Since the $S O(8)^{4}$ arises from Chan-Paton factors with constant gauge field background $a_{I}$, we apply the method developed in [31] [32] [6] to calculate type I one-loop threshold corrections. In this setup the expression for the one-loop amplitude of the open string reads

$$
\begin{gathered}
S_{1 \text {-loop }}^{I}=\frac{i}{2} V^{(8)} \sum_{\sigma=\mathcal{A}, \mathcal{M} ; i j} \rho_{\sigma} \int \frac{d t}{t} \frac{1}{\left(2 \pi^{2} t\right)^{4}} \sum_{a_{i j}^{\sigma}+\Gamma_{2}} e^{-\pi t p^{2} / 2} \frac{1}{\eta^{12}\left(\tau_{\sigma}\right)} \frac{i}{2} q_{i j}^{\sigma} B t \\
\frac{\theta_{1}^{\prime}\left(0, \tau_{\sigma}\right)}{\theta_{1}\left(\frac{i \epsilon_{\sigma} t}{2}, \tau_{\sigma}\right)} \sum_{\alpha} \frac{1}{2} s_{\alpha} \theta_{\alpha}\left(\frac{i \epsilon_{\sigma} t}{2}, \tau_{\sigma}\right) \theta_{\alpha}^{3}\left(0, \tau_{\sigma}\right)
\end{gathered}
$$

where $F=B Q$ is the background gauge field and $Q$ a generator of the Cartan subalgebra and $q_{i}$ the corresponding charge of the open string endpoint carrying Chan-Paton index i. The non-linear function $\epsilon_{\sigma}$ can be expanded as $\epsilon_{\sigma} \simeq q_{\sigma} B+\mathcal{O}\left(B^{3}\right)$ with $q_{i j}^{\mathcal{A}}=\left(q_{i}+q_{j}\right)$ and $q_{i j}^{\mathcal{M}}=2 q_{i}$.

Expanding the integrand to the order $\mathcal{O}\left(B^{4}\right)$ gives

$$
S_{1-\text { loop }}^{I}=-\frac{1}{2} V^{(8)} \sum_{\sigma=\mathcal{A}, \mathcal{M} ; i j} \rho_{\sigma} \int \frac{d t}{t}\left[\epsilon_{\sigma}^{4} \sum_{a_{i j}^{\sigma}+\Gamma_{2}} e^{-\pi t p_{I} G^{I J} p_{J} / 2}\right] .
$$

After Poisson resummation and changing variables from direct channel to closed string transverse channel which is $l=1 / t$ for the annulus and $l=1 /(4 t)$ for the Möbius strip, one finds:

$$
\begin{aligned}
S_{1-\text { loop }}^{I} & =-\frac{i}{2 \pi} V^{(8)} B^{4} \sum_{w} \frac{T_{2}}{w^{I} G_{I J} w^{J}}\left[\sum_{i j} e^{2 \pi i\left(a_{i}+a_{j}\right)_{I} w^{I}}\left(q_{i}+q_{j}\right)^{4}\right. \\
& \left.-\sum_{i} e^{4 \pi i a_{i I} w^{I}}\left(2 q_{i}\right)^{4}\right] .
\end{aligned}
$$


Evaluating the sum leads to

$$
\begin{aligned}
S_{1-\text { loop }}^{I} & =i \pi V^{(8)} B^{4}\left[4 \ln \left[T_{2} U_{2}|\eta(U)|^{4}\right] \sum_{i<j ;(i, j)=(k, k)}\left(q_{i}+q_{j}\right)^{4}\right. \\
& \left.+2 \sum_{k=2}^{4} \ln \left[T_{2} U_{2}\left|\frac{\theta_{k}(U)}{2 \eta(U)}\right|^{2}\right] \sum_{(i, j)=(\mathbf{1}, \mathbf{k})}\left(q_{i}+q_{j}\right)^{4}\right]
\end{aligned}
$$

where $k=\mathbf{1}, \ldots, \mathbf{4}$ and $\mathbf{1}=\{1, \ldots, 4\}, \mathbf{2}=\{5, \ldots, 8\}, \mathbf{3}=\{9, \ldots, 12\}, \mathbf{4}=\{13, \ldots, 16\}$. We omitted some moduli independent constant which appears after regularization of the logarithmic divergence [18].

In the T-dual type I' picture this example corresponds to placing four seven branes at each of the four fixed points. The above threshold corrections to $\operatorname{Tr} F_{S O(8)}^{2} \operatorname{Tr} F_{S O(8)_{k^{\prime}}}^{2}$ arise from open strings stretched between branes sitting on the same or different fixed points: e.g. for $k=k^{\prime}$ we have

$$
S_{1-\text { loop }}^{I}=i \pi V^{(8)} \ln \left[T_{2} U_{2}|\eta(U)|^{4}\right]\left(\operatorname{Tr} F_{S O(8)_{k}}^{2}\right)^{2} .
$$

On the heterotic side they translate to the degenerate orbit of the corresponding coupling.

\subsection{D-instanton Contribution}

Heterotic/type I duality maps winding modes of the heterotic string to winding modes of $D 1$-strings. Heterotic worldsheet instantons that arise in the non-degenerate orbit $A=$ $\left(\begin{array}{ll}k & j \\ 0 & p\end{array}\right)$ in the one loop amplitude, are 'dual' to BPS instantons which arise after wrapping the euclidean worldsheet of $D 1$ branes on the spacetime $T^{2}$. Choosing a basis of twocycles on $T^{2}$, all inequivalent ways in which a $T^{2}$ can cover $N$-times another $T^{2}$ are given by transformations that can be written in the form of the matrix $A$ with $k p=N$ and $0 \leq j<k$ and $p>0$ and $N$ is the instanton number. The embedding of the $D 1$-string worldsheet into the target space torus is then given by $X_{i}=A_{i j} \sigma^{j}$.

The classical instanton saddle point is the exponent of the Born-Infeld action of the wrapped $D 1$-brane [7] [8]:

$$
S_{B I}=\frac{1}{\lambda_{I}} \int d^{2} \sigma \sqrt{\operatorname{det}\left(\mathcal{G}_{I}+\mathcal{F}\right)}-i \int \mathcal{B}_{I}^{R}
$$

where $\mathcal{G}$ and $\mathcal{B}_{I}^{R}$ are the pull-backs of the metric and the antisymmetric tensor and $\mathcal{F}$ the $U(1)$ gauge background of the open string. 
Fluctuations around the classical instanton saddle point are described by the elliptic genus $\mathcal{A}(U)$ (and its descendents) for $N D$ 1-branes on $T^{2}$. Similarly we can take a single $D 1$-brane wrapped $N$ times over $T^{2}$. This is realized by the action of the Hecke operator on the elliptic genus $H_{N}[\mathcal{A}](\mathcal{U})$. The complex structure $U$ of the space-time $T^{2}$ is modified according to $\mathcal{U}=\frac{j+p U}{k}$ with the conditions for $j, p, k$ as above, e.g. the three inequivalent ways a torus can double cover another torus is $2 U, \frac{U}{2}$ and $\frac{1}{2}+\frac{U}{2}$.

Let us consider $\Delta_{R^{4} T^{4 g-4}}^{\text {non-deg }} \equiv I_{1}(g)$ from (2.42) $\left(\tilde{\lambda}_{1}=\tilde{\lambda}_{2}=\lambda\right)$ as an example, which in type I variables becomes

$$
\begin{aligned}
\left\langle R^{4} T^{4 g-4}\right\rangle_{\text {inst }} & =\frac{1}{(4 g) !} \frac{(-1)^{2 g-2}}{2^{4 g-5}} \frac{d^{4 g}}{d z^{4 g}} \sum \frac{1}{T_{2}^{4 g-3} k^{4 g-2}} \\
\times & \frac{[\sqrt{\operatorname{det} \mathcal{G}}-\sqrt{\operatorname{det}(\mathcal{G}+\mathcal{F}(z))}]^{4 g-4}}{\sqrt{\operatorname{det}(\mathcal{G}+\mathcal{F}(z))}} e^{-2 \pi S_{B I}} \mathcal{A}(\tilde{U}(z)),
\end{aligned}
$$

where

$$
\begin{aligned}
\tilde{U}(z) & =\frac{1}{k}\left[j+p U_{1}-i p U_{2} \sqrt{\operatorname{det}(\mathcal{G}+\mathcal{F}(z))} / \sqrt{\operatorname{det} \mathcal{G}}\right] \\
\sqrt{\operatorname{det}(\mathcal{G}+\mathcal{F}(z))} & =T_{2} k \sqrt{p^{2}-\frac{m z^{2}}{T_{2} U_{2}}} \\
\sqrt{\operatorname{det} \mathcal{G}} & =T_{2} k p \\
\mathcal{B}_{I}^{R} & =k p T_{1}
\end{aligned}
$$

and the skew symmetric eigenvalues of $\mathcal{F}$ are $f^{2}=-\frac{z^{2} k^{2} m T_{2}}{U_{2}}$.

In analogy to semiclassical instanton calculations the correlation function in an instanton background is obtained by saturating fermionic zero modes and integrating over the moduli space of instantons. In this case the instanton moduli space is provided by the heterotic matrix string model [33], which describes a worldsheet $O(N)$ two dimensional gauge theory. In the infrared limit this gauge theory flows to a $(8,0)$ supersymmetric $S_{N} \times \mathbf{Z}_{2}^{N}$ orbifold conformal field theory. The elliptic genus for $S_{N}$ symmetric orbifolds is naturally described by the action of the $N^{\prime}$ th Hecke operator on the elliptic genus [34]. It is known from [35] and [36] that the elliptic genus for $S_{N}$ symmetric orbifolds is given by the action of the $N^{\prime}$ th Hecke operator on the elliptic genus.

\subsection{One-Loop corrections to $R^{2}$}

Let us first briefly review threshold corrections in four dimensional $N=2$ string vacua, which are torus compactifications of six dimensional $N=1$ string vacua. In heterotic string 
vacua the Einstein term does not get any one loop corrections. This fact gives rise to a relation between threshold gauge couplings and the Kähler metric [37]. Since in type I theory Newton's constant is corrected at one loop [38] [39], the dilaton has to be redefined, which provides the one loop correction to the type I Kähler metric.

In eight dimensions this situation translates to the $R_{\mu \nu \rho \sigma} R^{\mu \nu \rho \sigma}$ coupling. There are no heterotic one-loop corrections, whereas in type I theory we will find a non-vanishing correction. The graviton vertex operator in the zero ghost picture is given in (3.6). Let us start with a two point amplitude. Although the kinematic structure of a two point amplitude vanishes due to the on shell constraints we can still calculate the four derivative gravitational coupling. Going to a three point amplitude which e.g. includes a modulus and two gravitons will give a non-vanishing kinematic structure and produce a derivative on the two point coupling with respect to the modulus.

The torus amplitude for a two point graviton vertex insertions vanishes since the fermionic zero modes cannot be saturated. But we can still get contributions from $\mathcal{K}$, $\mathcal{A}$ and $\mathcal{M}$. We want to extract the order $\mathcal{O}\left(k^{4}\right)$ term of the amplitude, which will only produce a non-vanishing result, if at least eight fermions are contracted due to Riemann identities.

For non-oriented surfaces contractions between chiral and anti-chiral fermions are allowed $\langle\psi(z) \bar{\psi}(\bar{w})\rangle_{\sigma}=G_{F}\left(z, I_{\sigma}(w)\right)$ with the involution $I_{\mathcal{A}}(w)=I_{\mathcal{M}}(w)=I_{\mathcal{K}}-\frac{\tau}{2}=$ $1-\bar{w}$ and $G_{F}(z, w)=\frac{i}{2} \frac{\theta_{\alpha}(z-w, \tau) \theta_{1}^{\prime}(\tau)}{\theta_{1}(z-w, \tau) \theta_{\alpha}(\tau)}$. Using (3.4) and taking the sum over worldsheets $\sigma=\mathcal{K}, \mathcal{A}, \mathcal{M}$ we finally obtain for the type I one loop $R^{2}$ correction

$$
\Delta_{\text {grav }}^{I}(U)=\frac{V^{8} T_{2}}{2^{8} \pi^{5}} \Gamma(3) c_{0} \frac{U_{2}^{3}}{T_{2}^{3}} \sum_{j, p \neq 0} \frac{1}{|j-p U|^{6}},
$$

where $c_{0}=\frac{N^{2}-N+2^{4}}{2}$. The coupling coincides with the one-loop correction to $F_{1}^{2} F_{2}^{2}$ in eq. (2.32). In the decompactification limit $\Delta_{\text {grav }}^{I}$ disappears, in agreement with heterotictype I duality in ten dimensions [3]. The heterotic tree-level $R^{2}$ term is 'dual' to the disc diagram on the type I side. Duality relates this term to a one-loop correction to $R^{2}$ on the heterotic side. Since such a term does not exist, we conclude that on the type I side it is a combination of $R^{2}$ and $\Delta_{F_{1}^{2} F_{2}^{2}}$, which corresponds to the heterotic $R^{2}$ term. In particular this combination is such that no one-loop correction to $R^{2}$ is predicted on the heterotic side. A similar observation was recently made with the $R^{2}$ correction in the duality of heterotic-type IIA 40].

Acknowledgements: We thank C. Angelantonj, D. Anselmi, I. Antoniadis, C. Bachas, W. Lerche, and N.P. Warner for helpful discussions. St. St. thanks École Polytechnique for the warm hospitality. 


\section{Appendix A. Regularization of world-sheet torus integrals}

The integral for the degenerate orbit of (2.15) and (2.39) takes the generic form:

$$
\begin{aligned}
I_{2} & =T_{2} \int_{0}^{\infty} \frac{d \tau_{2}}{\tau_{2}^{2+\alpha+\epsilon}} \sum_{(j, p) \neq(0,0)} \frac{d \tau_{2}}{\tau_{2}^{2+\alpha+\epsilon}} e^{-\frac{\pi T_{2}}{\tau_{2} U_{2}} b} e^{i \theta(j+\bar{U} p)} c(0) \\
& =c(0) \frac{U_{2}}{\pi}\left(\frac{U_{2}}{\pi T_{2}}\right)^{\alpha+\epsilon} \Gamma(1+\alpha+\epsilon) \sum_{(j, p) \neq(0,0)} \frac{e^{i \theta(j+\bar{U} p)}}{b^{1+\alpha+\epsilon}},
\end{aligned}
$$

with some:

$$
b=(j+B)^{2}+C^{2} .
$$

We have introduced a regularization $\epsilon$, which is necessary for $\alpha=0$. This also allows us to interchange the order of summation and integration. For the sum over $j$ we use the formula $(s \geq 1, C \neq 0)$ :

$$
\begin{aligned}
\sum_{j=-\infty}^{\infty} \frac{e^{i \theta j}}{\left[(j+B)^{2}+C^{2}\right]^{s}} & =\frac{2 \pi^{s}}{\Gamma(s)} \frac{|C|^{s-\frac{1}{2}}}{C^{2 s-1}} \sum_{r \neq 0} e^{2 \pi i\left(r-\frac{\theta}{2 \pi}\right) B}\left|r-\frac{\theta}{2 \pi}\right|^{s-\frac{1}{2}} K_{s-\frac{1}{2}}\left[2 \pi|C|\left|r-\frac{\theta}{2 \pi}\right|\right] \\
& + \begin{cases}\frac{2^{3 / 2-s} \pi^{1 / 2}}{\Gamma(s)} \frac{1}{C^{2 s-1}} e^{-i \theta B}|\theta C|^{s-1 / 2} K_{s-1 / 2}(|\theta C|), & \theta \neq 0 \\
\frac{\pi^{1 / 2}}{\Gamma(s)} \Gamma(s-1 / 2) \frac{1}{C^{2 s-1}}, & \theta=0\end{cases}
\end{aligned}
$$

Here $K_{\nu}(z)$ is the modified Bessel function (of the third kind)

$$
\begin{aligned}
K_{\nu}(z) & =\frac{1}{2} \Gamma(\nu) \Gamma(1-\nu)\left[I_{-\nu}(z)-I_{\nu}(z)\right] \\
I_{\nu}(z) & =\sum_{m=0}^{\infty} \frac{\left(\frac{z}{2}\right)^{2 m+\nu}}{m ! \Gamma(m+\nu+1)}
\end{aligned}
$$

while $I_{\nu}(z)$ being the modified Bessel function (of the first kind) [41]. For $s=1$ and $0 \leq \theta<2 \pi, C>0$ eq. (A.3) reduces to the Sommerfeld-Watson transformation. In fact, with $K_{\frac{1}{2}}(z)=\sqrt{\frac{\pi}{2 x}} e^{-x}$, we obtain:

$$
\begin{aligned}
& \sum_{j=-\infty}^{\infty} \frac{e^{i \theta j}}{(j+B)^{2}+C^{2}}= \\
& \quad \frac{\pi}{C}\left[e^{-i \theta(B-i C)}+e^{-i \theta(B+i C)} \frac{e^{2 \pi i(B+i C)}}{1-e^{2 \pi i(B+i C)}}+e^{-i \theta(B-i C)} \frac{e^{-2 \pi i(B-i C)}}{1-e^{-2 \pi i(B-i C)}}\right] .
\end{aligned}
$$

Our formula (A.3) may be obtained from a generalization of the identity (79) given in [42]. 
In the following we concentrate on the extra contribution to (A.1) originating from the regularization. It arises from the second term in (A.3), i.e. it is the contribution from $r=\frac{\theta}{2 \pi}$. We shall denote it by $I_{2}(\epsilon)$. The first term in eq. (A.3), given by a sum over integers $r$ and the Bessel function, has already been evaluated in section 2 and leads to the polylogarithms.

Now we come to the regularization of (2.15), which is necessary for $2 \alpha+N \equiv 2 s-$ $2 r+N=0$. In that case one has to replace the second sum in (2.26) with the expression:

$$
\begin{aligned}
I_{2}(\epsilon)=\frac{\partial^{N}}{\partial \Lambda_{1}^{q_{1}} \partial \Lambda_{2}^{q_{2}} \partial \Lambda_{3}^{q_{3}} \partial \Lambda_{4}^{q_{4}}} & \frac{2}{\left[1-i\left(\Lambda_{1}+\Lambda_{2}+\Lambda_{3}+\Lambda_{4}\right)-\frac{1}{4}\left(\Lambda_{1}-\Lambda_{2}+\Lambda_{3}-\Lambda_{4}\right)^{2}\right]^{\frac{1}{2}(1+2 \alpha+2 \epsilon)}} \\
& \times\left.\frac{(2 \pi)^{\alpha}}{\pi^{1 / 2}}\left(\frac{1}{\pi T_{2} U_{2}}\right)^{\epsilon} \Gamma\left(\frac{1}{2}+\alpha+\epsilon\right) \zeta(1+2 \epsilon)\right|_{\Lambda_{i}=0} .
\end{aligned}
$$

Next, the regularized expression for the $p$-sum in $(2.43)$ is $(k=2 g-2)$ :

$$
\begin{aligned}
\frac{2}{\left(2 T_{2} U_{2}\right)^{k}} & \sum_{\substack{\alpha=0 \\
\alpha=\alpha_{1}+\alpha_{2}}}^{2 g} \sum_{s=0}^{k}(-1)^{s-k} \pi^{-\alpha-\frac{1}{2}} 2^{-4 k+2 s+\alpha} \frac{(2 k) !}{(2 s) !(k-s) !}\left(\frac{1}{2 T_{2} U_{2}}\right)^{\alpha-k}\left(\frac{1}{\pi T_{2} U_{2}}\right)^{\epsilon} \\
& \times \zeta(1-2 k+2 \alpha+2 \epsilon) \Gamma\left(\frac{1}{2}+s-k+\alpha+\epsilon\right) c\left(0 ; 2 g-2 \alpha_{1}, 2 g-2 \alpha_{2}\right) \frac{(-\pi)^{\alpha}}{\left(\alpha_{1}\right) !\left(\alpha_{2}\right) !} .
\end{aligned}
$$

We have used the relations $\left(\nu=\frac{1}{2}+\alpha+\epsilon\right)$ :

$$
\begin{aligned}
\left.\frac{d^{2 s}}{d x^{2 s}} e^{-a x^{2}}\right|_{x=0} & =\frac{(2 s) !}{(s) !}(-a)^{s} \\
\left.\frac{d^{2 k}}{d x^{2 k}} e^{-x} x^{\nu} K_{\nu}(x)\right|_{x=0} & =\sum_{s=0}^{k}(-1)^{s-k} 2^{-2 k+2 s-\frac{1}{2}+\alpha+\epsilon} \frac{(2 k) !}{(2 s) !(k-s) !} \Gamma(\nu+s-k) .
\end{aligned}
$$

The sum (A.7) agrees with eq. (4.24) of [20] after performing some obvious redefinitions. For $\alpha=k=2 g-2$, we encounter

$$
\begin{aligned}
I_{2}(\epsilon) & =\frac{2}{\left(2 T_{2} U_{2}\right)^{k}} \sum_{k=\alpha_{1}+\alpha_{2}} \sum_{s=0}^{2 g-2}(-1)^{s-k} \pi^{-k-\frac{1}{2}} 2^{-3 k+2 s} \frac{(2 k) !}{(2 s) !(k-s) !} \\
& \times\left(\frac{1}{\pi T_{2} U_{2}}\right)^{\epsilon} \zeta(1+2 \epsilon) \Gamma\left(\frac{1}{2}+s+\epsilon\right) c\left(0 ; 2 g-2 \alpha_{1}, 2 g-2 \alpha_{2}\right) \frac{(-\pi)^{k}}{\left(\alpha_{1}\right) !\left(\alpha_{2}\right) !}
\end{aligned}
$$

which must replace the $p$-sum of (2.43), when it is divergent. 
To obtain from (A.6) and (A.9) the $\epsilon$-independent terms, we have to use the series (cf. also [20]):

$$
\begin{aligned}
\zeta(1+2 \epsilon) & =\frac{1}{2 \epsilon}+\gamma_{E}+\mathcal{O}(\epsilon) \\
\Gamma\left(\frac{1}{2}+s+\epsilon\right) & =\Gamma\left(\frac{1}{2}+s\right)\left[\psi\left(\frac{1}{2}+s\right)+\epsilon\right]+\mathcal{O}\left(\epsilon^{2}\right), \\
\left(\frac{1}{\pi T_{2} U_{2}}\right)^{\epsilon} & =1-\epsilon \ln \left(\pi T_{2} U_{2}\right)+\mathcal{O}\left(\epsilon^{2}\right) .
\end{aligned}
$$

Here $\gamma_{E}$ is the Euler-Mascheroni constant $\gamma_{E}$.

A special case of (A.6) appears for $N=\alpha=0$, which corresponds to the integral:

$$
\int_{\mathcal{F}} \frac{d^{2} \tau}{\tau_{2}} \sum_{\left(p_{L}, p_{R}\right)} q^{\frac{1}{2}\left|p_{L}\right|^{2}} \bar{q}^{\frac{1}{2}\left|p_{R}\right|^{2}}=\kappa-\ln |\eta(T)|^{4}|\eta(U)|^{4} .
$$

The integrand has an IR-divergence for $\tau_{2} \rightarrow \infty$ and thus has to be regularized. This results in an extra contribution, given by $\kappa$. In ref. [18], the integral (A.11) has been regularized by substracting the (non-modular invariant) field-theoretical part with the result $\kappa=\gamma_{E}-\ln (4 \pi)-\ln \left(T_{2} U_{2}\right)-1-\ln \left(\frac{2}{3 \sqrt{3}}\right)$.

On the other hand, from our regularization (A.1), we obtain:

$$
I_{2}(\epsilon)=\frac{2}{\pi^{\frac{1}{2}}}\left(\frac{1}{\pi T_{2} U_{2}}\right)^{\epsilon} \Gamma\left(\frac{1}{2}+\epsilon\right) \zeta(1+2 \epsilon) .
$$

Extracting from $I_{2}(\epsilon)$ the $\epsilon$-independent terms gives

$$
\kappa=\gamma_{E}-\ln (4 \pi)-\ln \left(T_{2} U_{2}\right)
$$




\section{References}

[1] E. Witten, Nucl. Phys. B 443 (1995) 85.

[2] J. Polchinski, E. Witten, Nucl. Phys. B 460(1996) 525.

[3] A. A. Tseytlin, Nucl. Phys. B 467 (1996) 383; Phys. Lett. B 367 (1996) 84

[4] C. Angelantonj, M. Bianchi, G. Pradisi, A. Sagnotti, Y. S. Stanev, Phys. Lett. B 385 (1996) 96

[5] M. Bianchi, G. Pradisi, A. Sagnotti, Nucl. Phys. B 376(1992) 365

[6] C. Bachas, E. Kiritsis, Nucl. Phys. Proc. Suppl. 55 B (1997) 194.

[7] C. Bachas, C. Fabre, E. Kiritsis, N.A. Obers, P. Vanhove, Nucl. Phys. B 509 (1998) 33.

[8] E. Kiritsis and N. Obers, J. High Energy Phys. 10 (1997) 4.

[9] M. Bianchi, E. Gava, F. Morales, K.S. Narain, Nucl. Phys. B 547 (1999) 96.

[10] W. Lerche and S. Stieberger, Adv. Theor. Math. Phys. 2 (1998) 1105.

[11] W. Lerche, S. Stieberger, N.P. Warner, Quartic Gauge Couplings from K3 Geometry, hep-th/9811228; Prepotentials from symmetric products, hep-th/9901162.

[12] L. Baulieu and S. Shatashvili, Duality from topological symmetry, hep-th/9811198.

[13] A. Salam and E. Sezgin, Phys. Lett. B 154 (1985) 37;

M. Awada and P. K. Townsend, Phys. Lett. B 156 (1985) 51.

[14] A. Schellekens, N.P. Warner, Phys. Lett. B 177 (1986) 317; Phys. Lett. B 181 (1986) 339; Nucl. Phys. B 287 (1987) 87; W. Lerche, B.E.W. Nilsson and A. N. Schellekens, Nucl. Phys. B 289 (1987) 609; W. Lerche, B.E.W. Nilsson, A.N. Schellekens and N.P. Warner, Nucl. Phys. B 299 (1988) 91; W. Lerche, A.N. Schellekens and N.P. Warner, Phys. Rept. 177 (1989) 1.

[15] W. Lerche, Nucl. Phys. B 308 (1988) 102.

[16] K. Pilch, A. Schellekens, N.P. Warner, Phys. Lett. B 287 (1987) 362

[17] M. B. Green, J.H. Schwarz, E. Witten, Superstring Theory,Cambridge U. Press

[18] L. Dixon, V. Kaplunovsky and J. Louis, Nucl. Phys. B 355 (1991) 649.

[19] K. Förger and S. Stieberger, Nucl. Phys. B 514 (1998) 135.

[20] M. Marino, G. Moore, Nucl. Phys. B 543 (1999) 592.

[21] I. Antondiadis, E. Gava, K. S. Narain, T. R. Taylor, Nucl. Phys. B 455 (1995) 109

[22] J. P. Serre, Cours d'Arithmetique, PUF Paris 1970

[23] A. Terras, Harmonic Analysis on Symmetric Spaces and Applications (1985) SpringerVerlag

[24] S. Stieberger, Nucl. Phys. B 541 (1999) 109.

[25] N. Berkovits and C. Vafa, Nucl. Phys. B 433 (1995) 123.

[26] S. Yau and E. Zaslow, BPS states, string duality, and nodal curves on K3, hepth/9512121; 
L. Göttsche, A conjectural generating function for numbers of curves on surfaces, alggeom/9711012;

L. Bryan and N. Leung, The enumerative geometry of K3 surfaces and modular forms, alg-geom/9711031.

[27] N. Berkovits and C. Vafa, Nucl. Phys. B 533 (1998) 181.

[28] M. Serone, Phys. Lett. B 395 (1997) 42; I. Antoniadis, H. Partouche, T. R. Taylor, Nucl. Phys. B 489 (1997) 160; J. F. Morales, M. Serone, Nucl. Phys. B 501 (1997) 427.

[29] E. Kiritsis and B. Pioline, Nucl. Phys. B 508 (1997) 509.

[30] A. Sen, Nucl. Phys. B 475 (1996) 562

[31] C. Bachas, M. Porrati, Phys. Lett. B 296 (1992) 77.

[32] C. Bachas, C. Fabre, Nucl. Phys. B 476 (1996) 418.

[33] D. A. Lowe, Phys. Lett. B 403 (1997) 243; P. Horava, Nucl. Phys. B 505 (1997) 84; S. J. Rey, Nucl. Phys. B 502 (1997) 170; D. Kabat, S. J. Rey, Nucl. Phys. B 508 (1997) 535 .

[34] R. Dijkgraaf, E. Verlinde, H. Verlinde, Nucl. Phys. B 500 (1997) 43.

[35] R. Dijkgraaf, G. Moore, E. Verlinde, H. Verlinde, Comm. Math. Phys. 185(1997) 197

[36] E. Gava, J. F. Morales, K. S. Narain, G. Thompson, Nucl. Phys. B 528 (1998) 95.

[37] I. Antondiadis, E. Gava, K. S. Narain, Nucl. Phys. B 383 (1992) 109; I. Antondiadis, E. Gava, K. S. Narain, T. R. Taylor, Nucl. Phys. B 407 (1993) 706

[38] I. Antoniadis, C. Bachas, C. Fabre, H. Partouche, T. R. Taylor, Nucl. Phys. B 489 (1997) 160

[39] I. Antoniadis, H. Partouche, T. R. Taylor, Nucl. Phys. Proc. Suppl. 61 A (1998) 58-71; Nucl. Phys. Proc. Suppl. 67 (1998) 3-16

[40] A. Gregori, C. Kounnas and P.M. Petropoulos, hep-th/9901117; hep-th/9904151.

[41] W. Magnus, F. Oberhettinger and F.G. Tricomi, Higher Transcendental Functions, volume 1 and 2, ed. A. Erdélyi, McGraw-Hill 1953.

[42] N. Vanegas-Arbeláez, Regularization of automorphic functions of manifolds with special Kähler geometry, hep-th/9906028. 